\title{
STRONGYLOIDES STERCORALIS AND ITS RISK FACTORS: AN EXPERIENCE AT A TERTIARY CARE HOSPITAL
}

\author{
Sunil Suresh Kuyare1, Avani Harkishen Koticha², Preeti Rajeev Mehta ${ }^{3}$ \\ ${ }^{1}$ Ex-Assistant Professor, Department of Microbiology, Seth G. S. Medical College and KEM Hospital. \\ 2Professor (Additional), Department of Microbiology, Seth G. S. Medical College and KEM Hospital. \\ 3 Professor and HOD, Department of Microbiology, Seth G. S. Medical College and KEM Hospital.
}

\begin{abstract}
Strongyloides stercoralis is one of the most neglected tropical parasitic infestations. The present study was carried out considering the paucity of data available from Indian context regarding the prevalence and risk factors associated with Strongyloides stercoralis infestation.

\section{METHODS}

The present study was an observational, retrospective audit/analysis where the hospital records of stool specimens received in Parasitology division for detection of intestinal parasites over a period of three years (January 2011 to December 2013) were analysed. The clinical features and laboratory data of patients infected with larvae of Strongyloides stercoralis were recorded. Descriptive statistics was applied to represent the categories of risk factors in percentages and chi-square test for its association with parasitic infection.
\end{abstract}

\section{RESULTS}

Larvae of Strongyloides stercoralis were detected in $0.4 \%(65 / 15530)$ stool specimens with a male preponderance. The most common presenting complaints by patients were attributed to diarrhoea 26/65 (40\%) and 14/65 (21.5\%) patients were asymptomatic. Peripheral eosinophilia was seen in 10.8\% (7/65) patients; $52 / 65$ (80\%) of patients were identified to have one or more of the known risk factors for Strongyloidiasis of which nearly half gave history of alcoholism (29/52,55.8\%); 3/65 (4.6\%) patients had hyperinfection of which one died. All three patients had two risk factors (alcoholism and steroids). Oral albendazole was used to treat the parasitic infestation in 31 (48.4\%), whereas $34(51.6 \%)$ received oral ivermectin.

\section{CONCLUSION}

The study findings of prevalence, clinical features and associated risk factors for Strongyloidiasis can be considered as preliminary report from Western Indian population. The presence of two risk factors in all cases of hyperinfection calls for further studies.

\section{KEYWORDS}

Strongyloides Stercoralis, Risk Factor, Alcoholism, Hyperinfection.

HOW TO CITE THIS ARTICLE: Kuyare SS, Koticha AH, Mehta PR. Strongyloides stercoralis and its risk factors: an experience at a tertiary care hospital. J. Evolution Med. Dent. Sci. 2016;5(50):3199-3202, DOI: 10.14260/jemds/2016/742

\section{INTRODUCTION}

Strongyloidiasis is a parasitic disease caused by a soil transmitted intestinal nematode, Strongyloides stercoralis. Filariform larvae of Strongyloides stercoralis penetrate the skin, undergo heart and lung migration, migrate into alveoli and subsequently ascend to trachea. Larvae are swallowed and develop into adult worms in small intestine. They produce eggs, which are deposited in intestinal mucosa where they hatch into rhabditiform larvae. This parasite is endemic to tropical and sub-tropical countries. Its existence in the immunocompromised patients is well known. The different immunocompromised patients infected with Strongyloides stercoralis reported are alcoholics, HIV sero positive, Systemic Lupus Erythematosus (SLE), Hansen's disease, etc.(1-3)

Financial or Other, Competing Interest: None.

Submission 21-05-2016, Peer Review 03-06-2016,

Acceptance 06-06-2016, Published 22-06-2016.

Corresponding Author:

Dr. Sunil Suresh Kuyare,

\#1701, 17th Floor,

UG-PG Hostel Building,

KEM Hospital Campus,

Parel, Mumbai-400012.

E-mail:drsunil77@gmail.com

DOI: $10.14260 /$ jemds $/ 2016 / 742$
Worldwide an estimate of 30 to 100 million people are found to be infested by Strongyloides stercoralis.(4) The prevalence of Strongyloides stercoralis ranges between 0.2 and $3.4 \%$ worldwide, while in India it is reported to be $2.6 \%$. (5-8) Overt Strongyloidiasis may be manifested with intestinal symptoms such as abdominal pain, intermittent or persistent diarrhoea associated with pulmonary (Cough, wheezing, chronic bronchitis) or cutaneous (Pruritus, urticaria) symptoms.(9) However, some cases of asymptomatic manifestations have also been published.(10) Hyperinfection and disseminated infections are always a risk in patients infected with Strongyloides stercoralis. The rhabditiform larvae may mature to filariform larvae, while still in bowel lumen which penetrate bowel wall to initiate a new cycle called autoinfection. Thus, this parasite has unique ability to replicate and increase in number without leaving its host permitting ongoing cycles of autoinfection and can thus persist for decades without further exposure of the host to exogenous infective larvae.(11)

Immunosuppressive conditions like HIV and HTLV infection, prolonged steroid use, alcoholism and cytotoxic drugs can accelerate autoinfectious cycle and can cause overwhelming replication of the parasite progressing to hyperinfection or dissemination.(8) Hence, the present study 
was carried out to estimate the prevalence and the risk factors associated with Strongyloides stercoralis.

\section{MATERIALS AND METHODS}

The present study was an observational, retrospective type of study, which was initiated after obtaining approval from the Institutional Ethics Committee and waiver for informed consent. The confidentiality of all the patients was strictly maintained. Stool specimens received in Parasitology Division, Department of Microbiology of a tertiary care public hospital for detection of intestinal parasites over a period of three years, i.e. January 2011 to December 2013 were analysed. As per the laboratory protocol, the stool specimens were routinely tested by saline mount and iodine mount as well as saturated salt solution technique as concentration method. The clinical and laboratory data of the patients whose stool specimens revealed larvae of Strongyloides stercoralis were analysed. The clinical data recorded was age, gender, chief presenting complaints, clinical diagnosis and presence of any known risk factors and administered anti-helminthic drug/s. The laboratory findings included haemoglobin value and presence of eosinophilia.

As a standard laboratory protocol, to rule out hyperinfection syndrome an additional sputum specimen of the patients infected with Strongyloidiasis was also submitted to Parasitology division. Two to three wet mounts were prepared from the sputum specimen received and were screened on low power and high power for presence of larvae of Strongyloides stercoralis. We also analysed the findings of sputum specimens in the present study. Post treatment, the findings of stool and sputum specimen from the patients was also analysed. Descriptive statistics was applied to represent the categories of risk factors in percentages. Chi-square test was used to assess the association of risk factors with the parasitic infestation.

\section{RESULTS}

\section{Demographic Details}

A single stool specimen from 15530 patients was received during the study period. Larva of Strongyloides stercoralis was detected in 65 stool specimens $(0.4 \%)$. The age of these infected patients ranged from 6 years to 65 years with the median (range) age of infested patients was 40 years. Of the 65 patients, 47 were males ( $72.3 \%$ ) and 18 were females (27.7\%) with male-to-female ratio of 2.6:1.

\section{Clinical Features of Patients Infected with Strongyloides Stercoralis}

Nearly one-fourth of the patients $(15 / 65,23.1 \%)$ were found to have alcoholic liver disease. Table 1 describes the clinical diagnoses of all the study patients infected with larvae of Strongyloides stercoralis. The most common presenting complaint by patients was diarrhoea $(26 / 65,40 \%)$ followed by cough and breathlessness $(20 / 65,31 \%)$. Two (3.1\%) patients had urticarial rash and three $(4.6 \%)$ patients had extreme fatigue. The remaining 14/65 (21.5\%) patients were asymptomatic. Peripheral eosinophilia was seen in $10.8 \%$ (7/65) patients. Larvae of Strongyloides stercoralis were detected in sputum specimen of $3 / 65(4.6 \%)$ patients. Of the three patients diagnosed to have hyperinfection syndrome, one succumbed to death.
Associated Risk Factors and Treatment of Patients Infected with Strongyloides Stercoralis

A total of 52/65 (80\%) of these patients were identified to have one or more of the known risk factors for Strongyloidiasis. Alcoholism was found in nearly half of the patients, i.e. $55.7 \%(29 / 52)$ followed by patients with prolonged use of steroids in $42.3 \%(22 / 52)$, while $21 \%$ $(11 / 52)$ patients were on immune-suppressants. Of the 52 patients with risk factors 43 patients had any one risk factor, 8 had any two risk factors, while only 1 had all the three risk factors. Three of the patients who were diagnosed with hyperinfection had two risk factors (alcoholism and use of steroids).

$31(48.4 \%)$ patients infected with Strongyloides stercoralis were treated with oral Albendazole, whereas 34 (51.6\%) received oral Ivermectin. Stool and sputum examination performed after 7 days of treatment did not reveal larvae of Strongyloides stercoralis in 64 of the 65 patients.

\begin{tabular}{|c|c|}
\hline Clinical Diagnosis & $\begin{array}{c}\text { Number of } \\
\text { Patients (\%) }\end{array}$ \\
\hline Alcoholic liver disease & $15(23.1)$ \\
\hline Anaemia & $8(12.3)$ \\
\hline $\begin{array}{l}\text { Chronic obstructive pulmonary disease } \\
\text { or bronchial asthma }\end{array}$ & $8(12.3)$ \\
\hline Routine health checkup & $7(10.8)$ \\
\hline Acute Gastroenteritis & $7(10.8)$ \\
\hline Nephrotic syndrome & $4(6.2)$ \\
\hline Systemic Lupus Erythematosus (SLE) & $4(6.2)$ \\
\hline HIV infection & $3(4.6)$ \\
\hline Hansen's disease & $3(4.6)$ \\
\hline $\begin{array}{l}\text { Cutaneous conditions } \\
\text { (Psoriasis/urticaria) }\end{array}$ & $2(3.1)$ \\
\hline Chronic Lymphoid Leukaemia & $1(1.5)$ \\
\hline Rheumatoid arthritis & $1(1.5)$ \\
\hline Diabetes Mellitus & $1(1.5)$ \\
\hline Abdominal tuberculosis & $1(1.5)$ \\
\hline \multicolumn{2}{|c|}{$\begin{array}{c}\text { Table 1: Clinical Diagnosis of the Patients Infected with } \\
\text { Larvae of Strongyloides Stercoralis }(n=65)\end{array}$} \\
\hline
\end{tabular}

\section{DISCUSSION}

The present study was undertaken to assess the prevalence of Strongyloides stercoralis infestation, associated clinical features and risk factors if any in a tertiary care public hospital from a developing country. We found out the prevalence of Strongyloidiasis to an extent of just $0.4 \%$ with a male preponderance. Four-fifths of the study patients (80\%) had one or more known risk factors, the most common being alcohol intake (55.8\%) followed by chronic use of corticosteroids (42.3\%). Three patients had hyperinfection, of which two had immunosuppressive factors and one died.

Strongyloides stercoralis infestation rates vary widely between studies due to the differences in the source of study participants (Hospital-based, refugee camps and community studies) and sensitivity of the diagnostic tests.(8) Different authors have used additional techniques like traditional Kato Katz technique, Hoffman-Pons-Janer technique, Koga agar plate technique, water emergence technique, Willis and Baermann-Moraes technique, Ludz method, etc. besides the 
saline mount and formol-ether sedimentation which improved the detection of Strongyloides stercoralis, thereby increase in the prevalence rate.(12-14) Our reports on the prevalence matches with reports from various parts of the world.(5-7) We also found out that a large number of infested patients were males corroborating a recent report by Azira et al.(15) More likelihood of alcohol consumption, eating behaviour and outside activities may be plausible reasons for the noted gender difference in the infestation rate.(15)

Alcohol consumption and immunosuppression either due to use of corticosteroids or immunosuppressants have been found to increase the risk of Strongyloidiasis.(1) The presence of alcoholism as a risk factor in $56 \%$ of the patients infected with Strongyloidiasis in the present study was found to be statistically significant ( $p=0.002$; Chi square test) when compared to steroid/immunosuppressant users. Schar et al conducted a meta-analysis of associated risk factor (alcohol) with Strongyloides stercoralis infestation and reported an odds ratio [95\% confidence interval] of 6.69 [1.47, 33.8].(8) Chronic alcoholics tend to have an increased exposure to the parasite and also tend to be malnourished. It is a known fact that the survival of Strongyloides stercoralis in chronic alcoholics is also due to the breakdown of immune responses in the gastrointestinal tract as a result of alteration in intestinal barriers and there is elevated cortisone, which suppresses the immunity further.(16) Also, alcohol stimulates the transformation of rhabditiform larvae to filariform larvae within the intestine, thereby leading to autoinfection.(17)

Strongyloides stercoralis is atypical in the helminthic group undergoing auto-infection, where the parasite undergoes reproduction within human beings leading to infection lasting for ages. Autoinfectivity is the reason for lifelong infestation of Strongyloides stercoralis as young larvae continue to be released from the adult worms and complete eradication of the adult worms only can result in permanent cure of the patient.(18) In fact, autoinfectivity can also lead to hyperinfection by the worm. Hyperinfection by Strongyloides stercoralis is mainly seen amongst immunocompromised individuals with a high case fatality rate of around $60 \%$. $^{(19-21)}$ The mortality rate of the patients having hyperinfection of the present study was $33.3 \%$.

This is characterized by an acceleration of the normal life cycle of Strongyloides stercoralis that increases its burden either in the skin or lungs or gastrointestinal tract. Such patients often present either with shock, disseminated intravascular coagulation, meningitis, renal failure or respiratory failure due to the worm load.(3) Another entity called disseminated Strongyloidiasis exists where the larvae spreads outside its path to other organs such as heart, liver, brain and urinary tract.(22) Fortunately, we did not have any patient with disseminated Strongyloidiasis in the present study. But the presence of two risk factors in all these hyperinfection cases calls for a further study to determine the risk of hyperinfection in patients with more than one risk factor.

A total of three out of 65 patients (4.6\%) in the present study had anaemia. Al-Zain did a cross-sectional analysis of 256 children in an African village and observed the prevalence of anaemia amongst Strongyloides stercoralis infested children to be $60 \%$ (Three out of five children).(5) Apart from a large proportion of children with low haemoglobin, the extent of haemoglobin reduction was maximum amongst these children in comparison to other parasitic infestations in the study.

The commonest complaint for the patients detected with Strongyloides stercoralis was diarrhoea followed by breathlessness. Surprisingly, nearly one-fifth of the patients in the present study were asymptomatic. These were the patients who had submitted their stool specimen as a part of routine medical examination. Five of these patients had no risk factors. However, they may be harbouring the parasite because of the previous infection which may have gone unnoticed or probably partially treated. Some of these asymptomatic patients had risk factors like alcoholism.(6) and use of steroid.(3) Thus, the present finding gives a hint that even asymptomatic patients with the presence of even one of the risk factor should be screened at regular intervals for presence of larvae of Strongyloides stercoralis.

The appearance of eosinophilia in only $10.8 \%$ of patients suggests that the eosinophilia is not so common in patients infected with Strongyloides stercoralis. Naidu et al in a recent study among refugees also concluded that eosinophilia has a poor predictability of Strongyloides stercoralis infestation. (23) Hence, diagnosis should be mainly based on combination of stool examination for the parasite and serology for the antibody.(24)

The study is limited by the following: Retrospective in nature with no details on the follow-ups of the patients; Diagnosis was based on a single stool specimen examination; Use of single technique for the detection of larvae of Strongyloides stercoralis and details on the amount and duration of alcohol consumption and dosage of corticosteroid were not assessed. However, the data from the present study can be considered as an initial report from Western India in reporting the prevalence, clinical features and associated risk factors for Strongyloides stercoralis.

\section{REFERENCES}

1. Keiser P, Nutman T. Strongyloides stercoralis in the immunocompromised population. Clin Microbiol Rev 2004;17(1):208-17.

2. Walzer P, Milder J, Banwell J, et al. Epidemiologic features of Strongyloides stercoralis infection in an endemic area of the united states. Am J Trop Med Hyg 1982;31(2): 313-9.

3. Asdamongkol N, Pornsuriyasak P, Sungkanuparph S. Risk factors for strongyloidiasis hyperinfection and clinical outcomes. Southeast Asian J Trop Med Public Health 2006;37(5):875-84.

4. Greaves D, Coggle S, Pollard C, et al. Strongyloides stercoralis infection. BMJ 2013;347:f4610.

5. Al-Zain B. Impact of socioeconomic conditions and parasitic infection on haemoglobin level among children in um-unnasser village, Gaza Strip. Turk J Med Sci 2009;39(1):53-8.

6. Abate A, Kibret B, Bekalu E, et al. Cross-sectional study on the prevalence of intestinal parasites and associated risk factors in teda health centre, northwest Ethiopia. ISRN Parasitology Article ID 757451, 2013;2013:1-5.

7. Chikukwa S, Lukas T. Prevalence and aetiological agents of parasitic infestation among population in northern Namibia. Int Sci Technol J Namibia 2015;5:97-103. 
8. Schar F, Trostdorf U, Giardina F, et al. Strongyloides stercoralis: global distribution and risk factors. PLoS Negl Trop Dis 2013;7(7):e2288.

9. Mokhlesi B, Shulzhenko O, Garimella PS, et al. Pulmonary strongyloidiasis: the varied clinical presentations. Clinical pulmonary medicine 2004;11(1):6-13.

10. Prendki V, Fenaux P, Durand R, et al. Strongyloidiasis in man 75 years after initial exposure. Emerg Infect Dis 2011;17(5):931-2.

11. Marques C, Zago-Gomes M, Goncalves C, et al. Alcoholism and strongyloides stercoralis daily ethanol ingestion has a positive correlation with the frequency of strongyloides larvae in the stools. PLoSNegl Trop Dis 2010;4(6):e717.

12. Carvalho G, Moreira L, Pena J, et al. A comparative study of the TF-Test $\AA$, kato-katz, hoffman-pons-janer, willis and baermann-moraes coprologic methods for the detection of human parasitosis. Mem Inst Oswaldo Crutz 2012;107(1):80-4.

13. Glinz D, Silué K, Knopp $S$, et al. Comparing diagnostic accuracy of kato-katz, koga agar plate, etherconcentration, and flotac for schistosoma mansoni and soil-transmitted helminths. PLoS Negl Trop Dis 2010;4(7):e754.

14. Getaneh A, Medhin G, Shimelis T. Cryptosporidium and Strongyloides stercoralis infections among people with and without HIV infection and efficiency of diagnostic methods for strongyloides in yirgalem hospital, southern Ethiopia. BMC Res Notes 2010;3:90.

15. Azira N, Rahman AM, Zeehaida M. Review of patients with Strongyloides stercoralis infestation in a tertiary teaching hospital, kelantan. Malays J Pathol 2013;35(1):71-6.
16. Teixeira $\mathrm{M}$, Inês $\mathrm{E}$, Pacheco $\mathrm{F}$, et al. Asymptomatic Strongyloides stercoralis hyper -infection in an alcoholic patient with intense anaemia. J Parasitol 2010;96(4): 833-5.

17. De Oliveira L, Ribeiro C, Mendes Dde M, et al. Frequency of strongyloides stercoralis infection in alcoholics. Mem Inst Oswaldo Cruz 2002;97(1):119-21.

18. Vadlamudi R, Chi D, Krishnaswamy G. Intestinal strongyloidiasis and hyperinfection syndrome. Clin Mol Allergy 2006;4:8.

19. Johnston F, Morris P, Speare R, et al. Strongyloidiasis: a review of the evidence for Australian practitioners. Aust J Rural Health 2005;13(4):247-54.

20. Soulsby H, Hewagama S, Brady S. Case series of four patients with strongyloides after occupational exposure. Med J Aust 2012;196(7):444.

21. Potter A, Stephens D, DeKeulenaer B. Strongyloides hyper-infection: a case for awareness. Ann Trop Med Parasitol 2003;97(8):855-60

22. Grover I, Davila R, Subramony C, et al. Strongyloides infection in a cardiac transplant recipient: making a case for pre-transplantation screening and treatment. Gastroenterol Hepatol (NY) 2011;7(11):763-6.

23. Naidu P, Yanow S, Kowalewska-Grochowska K. Eosinophilia: a poor predictor of strongyloides infection in refugees. Can J Infect Dis Med Microbiol 2013;24(2):93-6.

24. Requena-Mendez A, Chiodini P, Bisoffi Z, et al. The laboratory diagnosis and follow up of strongyloidiasis: a systematic review. PLoSNegl Trop Dis 2013;7(1):e2002. 